\title{
ON CLIFFORD'S THEOREM AND RAMIFICATION INDICES FOR SYMPLECTIC MODULES OVER A FINITE FIELD
}

\author{
by ROBERT W. VAN DER WAALL*
}

(Received 10th August, 1985)

\section{Introduction}

Let $K$ be a field, $G$ a finite group. Let $V$ be an (irreducible) $K G$-module, where $K G$ is the group algebra consisting of all formal sums $\sum_{g \in G} a_{g} g, a_{g} \in K, g \in G$. The action of $\alpha=\sum a_{g} g$ on an element $v \in V$ obeys the rule $v\left(\sum_{g \in G} a_{g} g\right)=\sum_{g \in G}\left(a_{g} v\right) g$. If $H$ is a subgroup of $G$, then, restricting the action of $G$ on $V$ to $H, V$ is also a $K H$-module. Notation: $V_{H}$.

Let now $N$ be a normal subgroup of $G$. The $K N$-module $V_{N}$ is not irreducible in general, even when $V$ is irreducible as $K G$-module. The well-known theorem of $\mathrm{A}$. $\mathrm{H}$. Clifford ([3], V.17.3) tells us precisely what is going on here.

Theorem (A. H. Clifford, 1938). Let $V$ be an irreducible KG-module. Let $N \triangleleft G$. Then the following properties hold.

(a) If $W$ is an irreducible $K N$-submodule of $V$, then $V=\sum_{g \in G} W g$. Every $W g$ is an irreducible $K N$-module and $V$ is a completely reducible $K N$-module.

(b) Let $W_{1}, \ldots, W_{n}$ be representatives of the isomorphism classes of the irreducible $K N$ submodules of $V$. Write

$$
V_{i}=\sum_{\substack{W \subseteq V \\ W \cong W_{i}}} W \quad(i=1, \ldots, n) .
$$

Then $V_{i}$ is homogeneous, i.e. it is a direct sum of $K N$-submodules of $V$, all being isomorphic to $W_{i}$, as $K N$-modules. Moreover $V=\bigoplus_{i=1}^{n} V_{i}$.

(c) Let $F_{i}$ be the irreducible representation of $N$ on $W_{i}$. Then $F_{i}^{g}$, defined by $\left(w_{i} g\right)\left(F_{i}^{q}(n)\right)=$ $\left(w_{i} F_{i}(n)\right) g, w_{i} \in W_{i}, g \in G$ is the irreducible representation of $N$ on $W_{i} g$.

(d) The homogeneous components $V_{i}$ of the $K N$-module $V$ are permuted transitively by elements of $G$ by multiplication on the right.

(e) For every $j$ the equality

$$
\left\{g \mid g \in G, V_{j} g=V_{j}\right\}=\left\{g \mid g \in G, F_{j}^{g} \text { equivalent to } F_{j}\right\}
$$

*This paper forms part of the Proceedings of the conference Groups-St Andrews 1985. 
holds. These elements $g$ constitute the subgroup $A_{j}$ (say) of $G$. Then $V_{j}$ is an irreducible $K A_{j}$-module. We have $V \cong V_{j} \bigotimes_{K A_{j}} K G=V_{j}^{G}$ ("V is induced by $V_{j}$ ").

(f) Let $D$ be the representation of $G$ on $V$. The irreducible constituents of $D_{N}$ are precisely all the $G$-conjugates $F^{g}$ of a single irreducible representation $F$ of $N$. They occur all with the same multiplicity $e$.

(g) If $\chi$ is the trace function of $D$ and if $\phi$ is the trace function of an irreducible constituent $F$ of $D_{N}$, then $\chi_{N}=e\left(\sum_{i=1}^{n} \phi^{g_{i}}\right)$, where the $g_{i}$ are representatives of the right cosets of the subgroup $A=\left\{g \mid g \in G, F^{g}\right.$ equivalent to $\left.F\right\}$ in $G$. Notice that $A \supseteq N$. The positive integer $e$ is called the inertia index (or ramification index) of $D$ (or $V$ ) over $N$.

Let $G, N$ and $A$ be the groups just mentioned in Clifford's Theorem. Sometimes we would like to know whether $e$ divides $|A / N|$. This happens certainly in two well known cases:

1. $K$ algebraically closed of charactertistic zero or of positive characteristic not dividing the order of $G$; see [13], page 35 .

2. $K$ a finite field of odd characteristic not dividing the order of $G$ and containing the primitive $m$ th-roots of unity, where $m=|G|_{2^{\prime}}, G / N$ an elementary abelian $p$ group; see [10], Theorem 13, due to W. Willems.

It is not true that the divisibility property of the inertia index always holds. As an example, take $R$ cyclic of order $3, K=\mathbb{F}_{2},\{1\}=N \Delta R$. Then there exists an irreducible two-dimensional $\mathbb{F}_{2}$-representation of $R$ with inertia index 2 over $N$. One of the purposes of this paper is to show that the behaviour of $e$ can be described if $G / N$ has prime order, $G$ arbitrary, $K$ a finite field. It is done in Theorem $\mathrm{E}$.

In this paper we also study the situation in which $\mathbb{F}$ is a finite field, $V$ a $\mathbb{F} G$-module, such that the vector space $V$ carries a non-singular alternating bilinear form with values in $\mathbb{F}$, which is left invariant by $G$. Such a $\mathbb{F} G$-module is called symplectic. If $L$ is a $\mathbb{F} G$ module, then $L^{*}$ will denote the dual module. Thus $L^{*}=\operatorname{Hom}_{\mathbb{F}}(L, \mathbb{F})$ and the action of $G$ on $L^{*}$ is defined by $v(\alpha g)=\left(v g^{-1}\right) \alpha$ for $\alpha \in L^{*}, g \in G, v \in L$. If $L \cong L^{*}$ as $\mathbb{F} G$-modules then $L$ is called self-dual. It is well known that $L$ is self-dual if and only if $L$ carries a nonsingular, G-invariant, bilinear form.

The following situations will be studied.

I. Let $\mathbb{F}$ be a finite field and let $V$ be a faithful irreducible symplectic $\mathbb{F} G$-module. Let $N \triangleleft G,|G / N|=$ odd prime number. What does the decomposition of $V_{N}$ look like? Or, what happens with $\left(V \bigotimes_{F} K\right)_{N}$ for a suitable field extension $K$ of finite degree over $\mathbb{F}$ ? Does an irreducible constituent of $V \bigotimes_{F} K$ decompose as a direct sum of irreducible $K N$-modules, each being symplectic and standing perpendicular to each other with respect to the (tensored) symplectic $K$-form? What about the ramification index $e$ ? Is it equal to 1 , to $|G / N|$, or to something else? An answer to these questions will be given in Theorem A. In a Corollary to Theorem A somewhat more can be said when $\mathbb{F}$ has characteristic 2 .

II. If we impose more conditions on the group $G$, then we can sharpen Theorem $A$. The result is Theorem B. The proof of Theorem B is a corollary to Theorem A. 
III. Suppose that the symplectic $\mathbb{F} G$-module $V$ with $\mathbb{F}$ a finite field, is a direct sum of pairwise non-isomorphic, self-dual, irreducible $\mathbb{F} G$-modules. We say that such a $\mathbb{F} G$ module is monoprimary. Let $N \triangleleft G$. Suppose that the order of $G / N$ is odd and assume that every prime divisor of $|G / N|$ divides $|\mathbb{F}|-1$. Then $V_{N}$ is monoprimary (Theorem $\mathrm{C}$ ). In order to prove that theorem we first consider the special case where $V$ is an irreducible symplectic $\mathbb{F} G$-module, $|G / N|=$ odd prime number $q, q$ divides $|\mathbb{F}|-1$. It turns out that $V_{N}$ is monoprimary and so the inertia index $e$ is equal to 1 (Theorem D). The statement of Theorem $D$ resembles that of the analogous statement made in the proof of Theorem (3.1) of [8]. The method of the proof of Theorem $\mathrm{D}$ given here, can be regarded as a specialization of the proof of Theorem A. For an application of Theorem $\mathrm{D}$ we refer to Theorem (2.3) of [12]. It shows that in Theorem $\mathrm{C}$ the word "monoprimary" can be replaced by the word "anisotropic". As such, (2.3) of [12] is a generalization of (3.1) of [8]. It then yields one of the main results of [12] stated as follows.

Theorem ([12], R. W. van der Waall and N. S. Hekster). Suppose that $p$ is an odd prime, that $G$ is a finite p-solvable group, that $N$ is a normal subgroup of $G$, and that $\chi$ is a monomial irreducible character of $N$ whose degree $\chi(1)$ is a power of $p$. Let $\eta$ be an irreducible constituent of the induced character $\chi^{G}$. Assume that every prime divisor of $|G / N|$ divides $p(p-1)$ and that $G / N$ is supersolvable of odd order. Then $\eta$ is a monomial character.

The above theorem should be compared with Dade's Theorem (0) in [2]:

Theorem ([2], E. C. Dade). Suppose that $p$ is an odd prime, that $G$ is a finite $p$ solvable group, that $\psi$ is a monomial irreducible character of $G$ whose degree $\psi(1)$ is a power of $p$, that $N$ is a subnormal subgroup of $G$, and that an irreducible character $\chi$ of $N$ is a constituent of the restriction $\psi_{N}$ of $\psi$. Then $\chi$ is monomial.

To conclude this Introduction, a few remarks are in order.

All the questions mentioned above about the inertia index $e$ and on the symplectic Schur-Clifford theory play an essential role in the (complex) representation theory of finite groups today. The reader is referred to papers of Isaacs, Berger, Dade, Parks and van der Waall; see notably $[1,2,6,7,8,9,10,11,12]$. In all these papers monomial characters are focussed as a central theme.

\section{Notations and conventions}

Most of the notations are standard and can be found in $[3,4,5]$ or are otherwise clear or self-explanatory. We recall some notions.

(1) Consider a type of operation on isomorphism classes of FG-modules (though apparently not in any natural way on the modules, themselves). We have in mind the following. Let $\alpha$ be an automorphism of $F$. If $V$ is an $F G$-module, then by a choice of basis, $V$ determines an $F$-representation $X$ of $G$. Application of $\alpha$ to the entries of the matrices $X(G)$ yields a new $F$-representation $X^{a}$. This corresponds to some $F G$-module 
whose isomorphism class is uniquely determined by $V$ and $\alpha$. We shall write $V^{\alpha}$ to denote any module in this class. If $F$ is a finite field with $b=p^{n}$ elements, with $p=\operatorname{char} F$, then $\operatorname{Gal}\left(F / \mathbb{F}_{p}\right)=\langle\beta\rangle$, where $\mathbb{F}_{p}$ is the prime field of $F$, and where $\beta$ is the Frobenius automorphism $x \mapsto x^{p}, x \in F$. We then denote $V^{\beta^{i}}$ sometimes by $V^{p^{i}}$.

(2) Definition (3.6) of [8]. Let $F \subseteq E$ be fields and let $V$ be an $E G$-module. Then $V$ is weakly self-dual over $F$ if $V^{*} \cong V^{\alpha}$ for some $\alpha \in \operatorname{Gal}(E / F)$.

(3) Lemma (3.4) of [8]. Let $N \triangleleft G$ with $G / N$ abelian and suppose that $F$ is a splitting field for $G / N$ with char $F$ not dividing $|G / N|$. If $V$ and $W$ are irreducible $F G$-modules such that $V_{N}$ and $W_{N}$ have a common irreducible constituent, then $W \cong V \mu$ for some linear $F$-character $\mu$ of $G / N$.

(4) Proposition (3.7) of [8]. Let $E \supseteq F$ be fields with $G a l(E / F)$ abelian, and let $V$ be an $E G$-module which is weakly self-dual over $F$. If $\lambda$ is an $F$-character of $G$ of odd multiplicative order and $V \lambda$ is also weakly self-dual over $F$, then $V \cong V \lambda$.

(5) $0_{2},(G)=$ product of all normal subgroups $M$ of $G$ with $2 \nmid M \mid$.

$F(G)=$ Fitting subgroup of $G$.

$\Omega_{1}(G)=\left\langle g \mid g \in G, g^{p}=1\right\rangle$; here $G$ is a $p$-group for some prime $p$.

$0_{p}(G)=$ the maximal normal $p$-subgroup of $G$.

$\mathbb{F}_{t}=$ finite field consisting of $t$ elements.

$\bar{E}=$ an algebraic closure of the field $E$.

$\mathbb{F}(\chi)$ : see the definition given in the last lines of page 151 of [5].

The theorems and their proofs

Theorem A. Let $G$ be a finite group. Suppose $V$ is a faithful irreducible non-singular symplectic $\mathbb{F} G$-module for a certain finite field $\mathbb{F}$. Let $N \triangleleft G,|G / N|=q$, where $q$ is an odd prime number. Then there exists a finite field $\mathbb{K}$ containing $\mathbb{F}$ such that at least one of the following properties holds.

(1) The $\mathbb{K} G$-module $V \bigotimes_{\mathbb{F}} \mathbb{K}$ contains a faithful irreducible non-singular symplectic $\mathbb{K} G$ module $W$ such that $W_{N}=U_{1} \perp \cdots \perp U_{q}$, where $U_{i} \not U_{j}$ as $\mathbb{K} N$-modules if $i \neq j$, the $U_{i}$ are irreducible non-singular symplectic $\mathbb{K} N$-submodules of $W_{N}$ for the symplectic form on $W$ restricted to $U_{i}$.

(2) The $\mathbb{K} G$-module $V \bigotimes_{\mathbb{F}} \mathbb{K}$ contains a faithful irreducible non-singular symplectic $\mathbb{K} G$ module $W$ such that $W$ is also irreducible when considered as $\mathbb{K} N$-module.

(3) There exists a self-dual absolutely irreducible $\mathbb{K} G$-module $T$ which is also absolutely irreducible as $\mathbb{K} N$-module and there exists a 2-dimensional irreducible $\mathbb{K} G$-module $S$ such that $N$ acts trivially on $S$ in such a way that $T \bigotimes_{K} S$ is isomorphic to a faithful irreducible non-singular symplectic $\mathbb{K} G$-submodule of $V \bigotimes_{\mathrm{F}} \mathbb{K}$.

Proof. There are two cases to be considered. Namely, (A) $V_{N}$ is not homogeneous, (B) $V_{N}$ is homogeneous.

(A) Let $V_{N}$ be not homogeneous. Then it follows from Clifford's theorem ([3], V.17.3) that $V_{N}$ is a direct sum of $q$ pairwise non-isomorphic $\mathbb{F} N$-submodules. Call them $U_{1}, \ldots, U_{q}$. Hence

$$
V_{N}=U_{1}+\cdots \dot{+} U_{q}
$$


In fact we see that here any irreducible $\mathbb{F} N$-submodule $T$ of $V_{N}$ is equal to precisely one of the $U_{i}$. With respect to the symplectic form it follows from a well known folklore theorem that the completely reducible $\mathbb{F} N$-module $V_{N}$ admits an orthogonal direct sum decomposition

$$
V_{N}=M_{1} \perp \cdots \perp M_{s} \perp\left(M_{s+1} \dot{+} M_{s+1}^{*}\right) \perp \cdots \perp\left(M_{s+t} \dot{+} M_{s+t}^{*}\right)
$$

where the $M_{1}, \ldots, M_{s}$ are irreducible non-singular symplectic $\mathbb{F} N$-modules with the form on $V$ restricted to $M_{i}$, and where all the $M_{s+1}, \ldots, M_{s+t}^{*}$ are irreducible totally isotropic $\mathbb{F} N$-modules; the matrix representation afforded by $M_{s+i}^{*}$ is the inverse-transpose to that afforded by $M_{s+i}$. Following the Krul-Schmidt Theorem applied on (1) and (2) there is at least one $U_{1}$ (say) exactly equal to some $M_{i}$ belonging to the set $\left\{M_{1}, \ldots, M_{s}\right\}$ as this set is not empty; namely $q=s+2 t$, again by the Krull-Schmidt Theorem as each of the $M_{1}, \ldots, M_{s+t}^{*}$ is its own homogeneous component in $V_{N}$. Write $M_{1}=U_{1}$. The $M_{1} g, g \in G$, are irreducible $\mathbb{F} N$-modules and they are all self-dual by construction of the action of $g$ on $V$. Thus $M_{1} g$ is precisely equal to one of the $M_{1}, \ldots, M_{s}$. Now, if $t$ would be an integer larger than zero, then we would conclude that $G$ does not act transitively on all the homogeneous components of $V_{N}$ by multiplication on the right. Clifford's Theorem, however, implies that $\left\{M_{1} g \mid g \in G\right\}$ is the set of the homogeneous components of $V_{N}$. Therefore $V_{N}=U_{1} \perp \cdots \perp U_{q}, U_{i} \supsetneqq U_{j}$ if $\neq j$. Hence $V_{N}$ is anisotropic in this case, i.e. $V_{N}$ does not contain isotropic $\mathbb{F} N$-submodules other than $(0)$.

(B) Let $b=p^{t}$ be the number of elements of $\mathbb{F}$, where $p=$ char $\mathbb{F}$. We now assume that $V_{N}$ is a direct sum of $e$ isomorphic irreducible $\mathbb{F} N$-submodules. Let $U$ be one of them. Set $V_{N}=e U$.

(B.1) Let $q=p$. Then Green's Theorem ([4], VII.9.19) yields $e=1, V_{N}=U$. Hence case (2) applies here with $\mathbb{K}=\mathbb{F}$.

(B.2) Let $q \neq p$. Then $[5,9.21]$ implies that

$$
V \bigotimes_{F} \bar{F}=\left(V_{1} \bigotimes_{K} \bar{F}\right) \dot{+}\left(V_{1}^{b} \bigotimes_{K} \bar{F}\right) \dot{+} \cdots \dot{+}\left(V_{1}^{b^{\alpha-1}} \bigotimes_{K} \bar{F}\right)
$$

where $\alpha=|\operatorname{Gal}(\mathbb{F}(\chi) / \mathbb{F})|$ and $K=\mathbb{F}(\chi)$, and where

$$
V \bigotimes_{F} K=V_{1} \dot{+} V_{1}^{b} \dot{+} \cdots \dot{+} v_{1}^{b^{\alpha-1}}
$$

(Notice that $K=\mathbb{F}\left(\chi^{b^{i}}\right)$, any $i=0, \ldots, \alpha-1$, by Theorem 9.21.c of [5].) Observe that $V_{1}^{b^{i}} ¥ V_{1}^{b^{j}}$ if $i \neq j$ and that the $V_{1}^{b^{i}}$ are absolutely irreducible $K G$-modules for any $i$ and that also

$$
V_{1}^{b^{i}} \bigotimes_{K} \bar{F} \not V_{1}^{b^{j}} \bigotimes_{K} \bar{F}
$$

if $i \neq j$. Now, if $S(\cdot, \cdot)$ is the symplectic form governing the $\mathbb{F} G$-module $V$, with values in $\mathbb{F}$, then $S_{1}(\cdot, \cdot)$ defined by

$$
S_{1}\left(\sum_{i}\left(x_{i} \otimes a_{i}\right), \sum_{j}\left(y_{j} \otimes b_{j}\right)\right)=\sum_{i, j} S\left(x_{i}, y_{j}\right) a_{i} b_{j}
$$


for all $\sum_{i}\left(x_{i} \otimes a_{i}\right), \sum_{j}\left(x_{j} \otimes b_{j}\right)$ in $V \bigotimes_{\mathrm{F}} K$, makes $V \bigotimes_{\mathrm{F}} K$ into a non-singular symplectic $K G$-module. As $V \bigotimes_{\mathrm{F}} K$ is completely reducible as $K G$-module, it follows again that an orthogonal direct sum decomposition holds as indicated,

$$
V \bigotimes_{F} K=M_{1} \perp \cdots \perp M_{a} \perp\left(M_{a+1} \dot{+} M_{a+1}^{*}\right) \perp \cdots \perp\left(M_{a+u} \dot{+} M_{a+u}^{*}\right) .
$$

Apply the Krull-Schmidt Theorem on (3) and (4). Then it follows that in (4) all the written $M$ 's are pairwise non-isomorphic and galois conjugated to each other.

(B.2. $\alpha$ ) Assume $u=0$, i.e. $V \bigotimes_{\mathbb{F}} K=M_{1} \perp \cdots \perp M_{a}$. Here $M_{1}$ is a faithful non-singular symplectic absolutely irreducible $K G$-submodule of $V \bigotimes_{F} K$. If $a \geqq 2$, then we apply induction to the dimension of the given irreducible module as vector space over its ground field and we conclude that the theorem holds. More precisely, replace $V$ by $M_{1}$ and $\mathbb{F}$ by $K$ in the statement of the theorem and observe that $M_{1} \otimes_{K} \mathbb{K}$ can be considered as $\mathbb{K} G$-submodule of $\left(V \bigotimes_{\mathbb{F}} K\right) \bigotimes_{K} \mathbb{K} \cong V \bigotimes_{K} \mathbb{K}$. Hence assume $a=1$. Then $V \bigotimes_{\mathbb{F}} \mathbb{F} \cong M_{1} \bigotimes_{K} \mathbb{F}$ is irreducible and so $V$ is an absolutely irreducible $\mathbb{F} G$-module. Hence

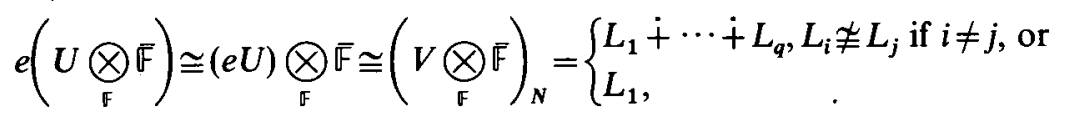

where the $L_{j}$ are the irreducible constituents of $\left(V \bigotimes_{\mathbb{F}} \overline{\mathbb{F}}\right)_{N}$; here we made use of Theorem VII.9.18 of [4], applied to the cyclic $p^{\prime}$-group $G / N$ of order $q$. Therefore certainly $e=1$ and we are in case (2) with $\mathbb{K}=\mathbb{F}$.

(B.2. $\beta$ ) Let $u \geqq 1$. Since $\left(V_{1}^{*}\right)^{b^{i}} \cong\left(V_{1}^{b^{i}}\right)^{*}$ for any $i$ and since $M_{t} \cong M_{i}^{*}$ if $t \in\{1, \ldots, a\}$, it cannot happen that $a \geqq 1$. Indeed, let $V_{1}=M_{1}$. Then for some $j, V_{1}^{b j}=M_{a+1} \supsetneqq M_{a+1}^{*}=$ $\left(V_{1}^{b^{j}}\right)^{*} \cong\left(V_{1}^{*}\right)^{b^{j}} \cong V_{1}^{b^{j}}$, with contradiction. Therefore we have

$$
V \bigotimes_{F} K=\left(M_{1} \dot{+} M_{1}^{*}\right) \perp \cdots \perp\left(M_{u} \dot{+} M_{u}^{*}\right) .
$$

Now $M_{1}^{*} \cong M_{1}^{r}$ for some $r=b^{f}$ with $f \in\{1, \ldots, 2 u-1\}$. Consider a matrix representation corresponding to the action of $G$ on $M_{1}$. Let $\omega_{1}, \ldots, \omega_{s}$, be the eigenvalues (counted with multiplicities, i.e. the representation is $s$-dimensional) of a matrix corresponding to a particular element $g \neq 1$ of $G$. Then $\omega_{1}^{-1}, \ldots, \omega_{s}^{-1}$ are the eigenvalues for the inversetranspose matrix corresponding to the element $g$. Therefore

$$
\sum_{i=1}^{s} \omega_{i}^{r}=\sum_{i=1}^{s} \omega_{i}^{-1}
$$

and also $\omega_{\sigma(i)}^{-1}=\omega_{i}^{r}, i=1, \ldots, s$ for some $\sigma$ contained in the symmetric group $\sum_{s}$. This leads to

$$
\begin{aligned}
\left(\sum_{i=1}^{s} \omega_{i}\right)^{r^{2}} & =\left(\sum_{i=1}^{s} \omega_{i}^{r}\right)^{r}=\left(\sum_{i=1}^{s} \omega_{i}^{-1}\right)^{r}=\sum_{i=1}^{s} \omega_{i}^{-r}=\sum_{i=1}^{s}\left(\omega_{i}^{r}\right)^{-1} \\
& =\sum_{i=1}^{s}\left(\omega_{\sigma(i)}^{-1}\right)^{-1}=\sum_{i=1}^{s} \omega_{\sigma(i)}=\sum_{i=1}^{s} \omega_{i} .
\end{aligned}
$$


Since $K=\mathbb{F}(\chi)$, it follows that $\sum_{i=1}^{s} \omega_{i} \in K \cap \mathbb{F}_{r^{2}} \subset \bar{F}$. This holds for all such traces and so $K \subseteq \mathbb{F}_{r^{2}}$. Moreover $M_{1}^{r^{2}} \cong M_{1}$, but $M_{1}^{r} \cong M_{1}^{*} ¥ M_{1}$. Certainly $r^{2} \in\left\{b^{2 u}, b^{4 u}, b^{6 u}, \ldots\right\}$. As now $r=b^{f} \leqq b^{2 u-1}<b^{2 u}$, we see that $r=b^{f}=b^{u}$ and so $K=\mathbb{F}_{r^{2}}$.

Thus we have $V \bigotimes_{\mathbb{F}} \mathbb{F}_{r}=L_{1}+\ldots+L_{u}, L_{i} \not L_{j}$ if $i \neq j$, and the $L_{i}$ are irreducible $\mathbb{F}_{r} G$ modules. It is clear that a numbering of the $L_{1}, \ldots, L_{u}$ can be chosen such that $L_{i} \bigotimes_{F_{r}} K \cong M_{i} \dot{+} M_{i}^{r} \cong M_{i} \dot{+} M_{i}^{*}, \quad i=1, \ldots, u$. Because of $\left(L_{i} \bigotimes_{F_{r}} K\right)^{*} \cong\left(M_{i} \dot{+} M_{i}^{*}\right)^{*} \cong$ $M_{i}^{*}+M_{i} \cong L_{i} \bigotimes_{\mathrm{Fr}} K,[4, \mathrm{VII} .8 .4]$ and $[5,9.7]$ imply that any $L_{i}$ is self-dual. By [4, VII.8.10.b] and the theorem of Krull-Schmidt we conclude that any $L_{i}$ is a non-singular faithful irreducible symplectic $\mathbb{F}_{r} G$-submodule of $V \bigotimes_{F} \mathbb{F}_{r}$ for the symplectic form on $V \bigotimes_{\mathbb{F}} \mathbb{F}_{r}$. Hence $V \bigotimes_{\mathbb{F}} \mathbb{F}_{r}=L_{1} \perp \cdots \perp L_{u}$; here it is also used that $V \bigotimes_{F} \mathbb{F}_{r}$ is completely reducible as $\mathbb{F}_{r} G$-module.

Now, if $u>1$, then we can apply induction just as we did it in the case (B.2. $\alpha$ ). Therefore, assume from now on that $u=1$. Hence $V \bigotimes_{F} K=M_{1}+M_{1}^{*}$. Thus $K=\mathbb{F}_{r^{2}}=\mathbb{F}_{b^{2}}$ and the $M_{1}$ and $M_{1}^{*}$ are non-isomorphic absolutely irreducible $\mathbb{F}_{b 2} G$-modules. It follows from Corollary 9.7 of [5] that the irreducible $\bar{F} G$-modules $M_{1} \bigotimes_{K} \overline{\mathbb{F}}$ and $M_{1}^{*} \bigotimes_{K} \bar{F}$ are not isomorphic. As $G / N$ is cyclic of prime order $q$ not equal to $p$, we see that either $\left(M_{1} \bigotimes_{K} \overline{\mathbb{F}}\right)_{N}$ is an irreducible $\overline{\mathbb{F}} N$-module (whence $\left(M_{1}^{*} \bigotimes_{K} \overline{\mathbb{F}}\right)_{N}$ is irreducible as well), or

$$
\left(M_{1} \bigotimes_{K} \overline{\mathbb{F}}\right)_{N}=T_{1} \dot{+} \cdots \dot{+} T_{q}, T_{j} \not T_{m} \text { if } j \neq m,
$$

where the $T_{i}$ are irreducible $\bar{F} N$-modules (whence $\left(M_{1}^{*} \bigotimes_{K} \bar{F}\right)_{N}$ decomposes in an analogous way), see Theorem VII.9.18 of [4]. In the very last case it follows that $T_{i}^{G} \cong M_{1} \bigotimes_{K} \overline{\mathbb{F}} \varsubsetneqq M_{1}^{*} \bigotimes_{K} \overline{\mathbb{F}} \cong\left(M_{1} \bigotimes_{K} \overline{\mathbb{F}}\right)^{*} \cong\left(T_{i}^{G}\right)^{*} \cong\left(T_{i}^{*}\right)^{G}$, whence all irreducible $\overline{\mathbb{F}} N$ modules contained in both $\left(M_{1} \bigotimes_{K} \bar{F}\right)_{N}$ and $\left(M_{1}^{*} \bigotimes_{K} \bar{F}\right)_{N}$ are pairwise non-isomorphic by the theorem of Frobenius-Nakayama. In that case we find

$$
\begin{aligned}
(V \underbrace{}_{\mathbb{F}} \overline{\mathbb{F}})_{N} & \cong\left(\left(M_{1}+M_{1}^{*}\right) \bigotimes_{K} \overline{\mathbb{F}}\right)_{N} \cong\left(\sum_{i=1}^{q} T_{i}+\sum_{i=1}^{q} T_{i}^{*}\right) \\
& \cong(e U) \underset{\mathbb{F}}{\bigotimes} \overline{\mathbb{F}} \cong e(U \underset{F}{\otimes} \bar{F}) .
\end{aligned}
$$

The Krull-Schmidt Theorem implies now that $e=1$, and so case (2) has been arrived at. Therefore we can assume that $\left(M_{1} \bigotimes_{K} \mathbb{F}\right)_{N}$ and $\left(M_{1}^{*} \bigotimes_{K} \mathbb{F}\right)_{N}$ remain irreducible as $\mathbb{F} N$ modules. This leads to

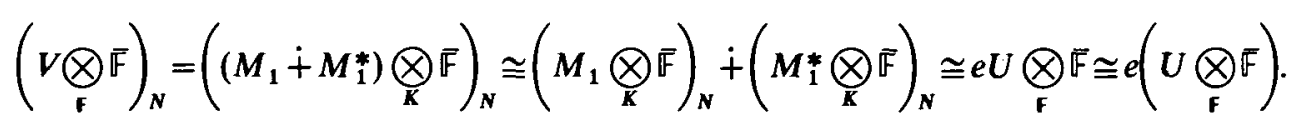

Applying the Krull-Schmidt Theorem we conclude that $e=1$ or $e=2$. Henceforth we are in case (2), or, as we will assume from now on, $e=2$. Write $M$ instead of $M_{1}$.

Under that assumption it is clear from the above, that $U$ is an absolutely irreducible F $N$-module. Hence $U \bigotimes_{\mathrm{F}} K$ is an absolutely irreducible $K N$-module. We have also $\left.M_{N} \cong M^{*}\right|_{N} \cong U \bigotimes_{\mathrm{F}} K$. We will show now that there exists an absolutely irreducible $\mathbb{F} G$ module $T$ such that $T_{N} \cong U$. Namely, if follows from Theorem VII.9.13 of [4] that any 
irreducible $K G$-module $L$ having $U \bigotimes_{\mathrm{F}} K$ in its restriction to $N$ (i.e. $L_{N}=U_{1} \dot{+} \cdots$ for a certain $K N$-submodule $U_{1}$ of $L$ with $U_{1} \cong U \bigotimes_{F} K$ ) is of the form $M \bigotimes_{K} \Lambda$, where $\Lambda$ is a one-dimensional $K G$-module such that $N$ acts trivially on $\Lambda$. Call $\lambda$ the corresponding one-dimensional representation of $G$. Let $\langle g N\rangle=G / N$. As $\left.M_{N} \cong M^{*}\right|_{N} \cong U \bigotimes_{\mathrm{F}} K$, it therefore holds that $M^{*} \cong M \bigotimes_{K} \Lambda$, where $\lambda\left(g^{i} n\right)=\omega^{i}$, any $n \in N$, with $\omega$ a certain primitive $q$ th-root of unity of $K$. Notice that $q \mid r^{2}-1$ but $q \Varangle r-1$, whence $q \mid r+1$. (Indeed, as $M \supsetneqq M^{*}$, some element $a=g^{j} n \in G \backslash N$ has $\operatorname{Tr} D(a) \neq 0$, where $\operatorname{Tr}$ means the trace function of the (matrix) representation $D$ which corresponds to the $K G$-module $M$; likewise we denote $D^{*}$ with respect to $M^{*}$. The fact that there must be such an element $a$ in $G \backslash N$ is just forced by $M^{*} \cong M \bigotimes_{K} \Lambda$ and $\left.M_{N} \cong M^{*}\right|_{N}$. So $\operatorname{Tr} D^{*}(a)=(\operatorname{Tr} D(a))^{r}=$ $(\operatorname{Tr} D(a)) \omega^{j}$, whence $\operatorname{Tr} D(a)=(\operatorname{Tr} D(a))^{r^{2}}=(\operatorname{Tr} D(a))^{r} \omega^{j r}=(\operatorname{Tr} D(a)) \omega^{j(1+r)}$, so that $\omega^{1+r}=1$. Thus if $q \mid r-1$, then $\omega^{2}=1=\omega^{q}$, whence $\omega=1$, a contradiction.)

Thus we have $\operatorname{Tr} D^{*}\left(g^{i} n\right)=\left(\operatorname{Tr} D\left(g^{i} n\right)\right)^{r}=\omega^{i}\left(\operatorname{Tr} D\left(g^{i} n\right)\right)$. Let $\Lambda^{h}$ be the one-dimensional $K G$-module corresponding to the representation $\lambda^{h}$ defined by $\lambda^{h}\left(g^{i} n\right)=\omega^{i h}$ for all $n \in N$. Hence $\lambda^{h}\left(g^{i} n\right)=\left(\lambda\left(g^{i} n\right)\right)^{h}$. Consider the irreducible $K G$-module $M \bigotimes_{K} \Lambda^{(q+1) / 2}$. Then $M \bigotimes_{K} \Lambda^{(q+1) / 2}$ is a self-dual $K G$-module, as we will show using the trace function. Indeed,

$$
\begin{aligned}
\operatorname{Tr}\left(\left(D \otimes \lambda^{(q+1) / 2}\right)^{*}\left(g^{i} n\right)\right) & =\omega^{-i(q+1) / 2}\left(\operatorname{Tr} D^{*}\left(g^{i} n\right)\right)=\omega^{-i(q+1) / 2} \omega^{i}\left(\operatorname{Tr} D\left(g^{i} n\right)\right) \\
& =\omega^{i(q+1) / 2}\left(\operatorname{Tr} D\left(g^{i} n\right)\right)=\operatorname{Tr}\left(\left(D \otimes \lambda^{(q+1) / 2}\right)\left(g^{i} n\right)\right)
\end{aligned}
$$

Even more, as $\omega^{r}=\omega^{-1}$ by $q \mid r+1$,

$$
\begin{aligned}
\left(\operatorname{Tr}\left(\left(D \otimes \lambda^{(q+1) / 2}\right)\left(g^{i} n\right)\right)\right)^{r} & =\omega^{i r(q+1) / 2}\left(\operatorname{Tr} D\left(g^{i} n\right)\right)^{r} \\
& =\omega^{-i(q+1) / 2}\left(\operatorname{Tr} D^{*}\left(g^{i} n\right)\right)=\omega^{-i(q+1) / 2} \omega^{i}\left(\operatorname{Tr} D\left(g^{i} n\right)\right) \\
& =\omega^{-i(q-1) / 2}\left(\operatorname{Tr} D\left(g^{i} n\right)\right) \\
& =\omega^{i(q+1) / 2}\left(\operatorname{Tr} D\left(g^{i} n\right)\right)=\operatorname{Tr}\left(\left(D \otimes \lambda^{q+1) / 2}\right)\left(g^{i} n\right)\right) .
\end{aligned}
$$

Therefore, Theorem VII.1.17 of [4] yields that $M \bigotimes_{K} \Lambda^{(q+1) / 2}$ can be realized over $\mathbb{F}$. This $M \bigotimes_{K} \Lambda^{(q+1) / 2}$ is now the desired $\mathbb{F} G$-module $T$ in case (3) as we will see.

The map $f$, defined by

$$
g^{i} n \stackrel{s}{\mapsto}\left(\begin{array}{cc}
0 & -1 \\
1 & \omega^{-(q-1) / 2}+\omega^{(q-1) / 2}
\end{array}\right)^{i}, \quad \text { for all } n \in N
$$

is a representation of $G$ to $S L(2, \mathbb{F})$ with $\operatorname{Ker} f=N$. The representation $f$ is irreducible as F-representation; namely the eigenvalues of

$$
\left(\begin{array}{cc}
0 & -1 \\
1 & \omega^{-(q-1) / 2}+\omega^{(q-1) / 2}
\end{array}\right)
$$

are $\omega^{-(q-1) / 2}$ and $\omega^{(q-1) / 2}$, both contained in $K$, but not in $\mathbb{F}$. 
Let $S$ be the $\mathbb{F} G$-module corresponding to $f$. Consider the $\mathbb{F} G$-module $T \bigotimes_{\mathrm{f}} S$. Then

$$
\begin{aligned}
\operatorname{Tr}\left(\left(D \otimes \lambda^{(q+1) / 2} \otimes f\right)\left(g^{i} n\right)\right) & =\operatorname{Tr}\left(D\left(g^{i} n\right) \otimes \lambda^{(q+1) / 2}\left(g^{i} n\right) \otimes f\left(g^{i} n\right)\right) \\
& =\left(\operatorname{Tr} D\left(g^{i} n\right)\right) \omega^{i(q+1) / 2}\left(\omega^{-i(q-1) / 2}+\omega^{i(q-1) / 2}\right) \\
& =\left(\operatorname{Tr} D\left(g^{i} n\right)\right) \omega^{i(1+q)}=\left(\operatorname{Tr} D\left(g^{i} n\right)\right)\left(\omega^{i}+1\right) \\
& =\operatorname{Tr} D^{*}\left(g^{i} n\right)+\operatorname{Tr} D\left(g^{i} n\right)
\end{aligned}
$$

Hence we see that the irreducible $\mathbb{F} G$-module $V$ (or rather the $K G$-module $V \bigotimes_{\mathrm{F}} K=$ $\left.M+M^{*}\right)$ and the $F G$-module $T \otimes_{\mathrm{F}} S$ afford the same trace function and that they have the same $\mathbb{F}$-dimension. Then Corollary 9.22 of [5] gives the result that $V$ and $T \otimes_{F} S$ are isomorphic as $\mathbb{F} G$-modules. Now, as

$$
T^{*} \bigotimes_{F} K \cong\left(T \bigotimes_{\mathbb{F}} K\right)^{*} \cong\left(M \bigotimes_{K} \Lambda^{(q+1) / 2}\right)^{*} \cong M \bigotimes_{K} \Lambda^{(q+1) / 2} \cong T \bigotimes_{F} K
$$

as $K G$-modules, it follows from the Deuring-Noether Theorem 9.7 of [5], that $T^{*} \cong T$ as $\mathbb{F} G$-modules. Hence we are in case (3).

In the characteristic 2 case of Theorem $A$, we can say a bit more.

Corollary to Theorem A. Let $G$ be a finite group. Assume that $N \triangleleft G$ with $|G / N|=$ odd prime $q$, and there is no $B \unlhd \neg$ with $B N=G$ and $B \cap N=\{1\}$. Suppose there exists $a$ faithful irreducible non-singular symplectic $\mathbb{F} G$-module $V$ where $\mathbb{F}$ is a finite field of characteristic 2. Then there exists a finite field $L \supseteq \mathbb{F}$ and a faithful irreducible non-singular symplectic $L G$-module $M$ such that

either

$M_{N}=U_{1} \perp \cdots \perp U_{q}$, where $U_{i} \not U_{j}$ as $L N$-modules if $i \neq j$, the $U_{i}$ are irreducible nonsingular symplectic $L N$-submodules of $M_{N}$,

or

$M_{N}$ is a faithful irreducible non-singular symplectic $L N$-module.

Proof. By assumption, $N \neq\{1\}$. Without loss of generality we may assume that we are in case (3) of Theorem A. Using the notation of that theorem, it follows that $T_{N}$ is not an irreducible $\mathbb{K} N$-module for the trivial representation of $N$. Hence $T$ is not the trivial $\mathbb{K} G$-module. Then, using char $\mathbb{K}=2$, a theorem of Fong([4], VII.8.13) implies that there exists a non-singular $G$-invariant symplectic form on $T$. As $N$ is trivially represented on $S$ and as $T \bigotimes_{K} S$ is a faithful $K G$-module, if follows from case (3) of Theorem $A$ that $T_{N}$ is faithful. Now, if $T$ would not be faithful as a $\mathbb{K} G$-module, we should have the existence of $\{1\} \neq B \triangleleft G$ with $B \cap N=\{1\}$, whence $B N=G$. This is contrary to our assumption. Hence $T$ is a faithful $\mathbb{K} G$-module. Certainly $\operatorname{dim}_{K} T \leqq \frac{1}{2} \operatorname{dim}_{\mathrm{f}} V$. So we have an induction machine with respect to the dimensions of the appropriate modules over their ground fields. The corollary now follows. 
Theorem B. Let $G$ and $V$ satisfy the hypotheses of Theorem $A$. Assume that $O_{2},(F(N)) \neq$ $\{1\}$ and that $N / F(N)$ is of odd order. Then case (3). of Theorem $A$ never occurs.

Proof. In the course of the proof of Theorem $A$ we used an induction argument without specifying, at that time, what in fact the induction step was! Therefore it is enough to show that we have a contradiction as soon as we have reached the point in the proof of Theorem $\mathrm{A}$, where we made the assumption that $e=2$. We proceed then as follows.

Hence it is clear that $U$ is an absolutely irreducible $\mathbb{F} N$-module. Moreover, as $U \bigotimes_{\mathrm{F}} \bar{F} \cong U^{*} \bigotimes_{\mathbb{F}} \bar{F}$, see above, it follows that the inverse-transpose representation $A^{*}$ of $N$ corresponding to $\left(U \bigotimes_{\mathrm{F}} \bar{F}\right)^{*}$ is $\bar{F}$-equivalent to the representation $A$ of $N$ on $U \bigotimes_{\mathrm{F}} \mathbb{F}$. Consider a representing matrix $A(n)$ with $n \in N$. Then, if $\omega \in \mathbb{F}$ is an eigenvalue of $A(n)$, the above conclusion implies that $\omega^{-1}$ is also an eigenvalue of $A(n)$. As $G$ is represented irreducibly and faithfully on $V$, a module of characteristic $p$, it follows that $O_{p}(G)$ is contained in the (trivial) kernel of the representation of $G$ on $V$, whence $O_{p}(G)=\{1\}$, see [3, V.5.17]. Therefore $\{1\} \neq B:=\Omega_{1}\left(O_{t}\left(Z\left(O_{2} \cdot(F(N))\right)\right)\right)$ for a certain odd prime $t$ unequal to $p$, by the hypothesis $O_{2},(F(N)) \neq\{1\}$. Hence $B$ is a non-trivial elementary abelian $t$ group with $B \triangleleft G$, and $B$ is not contained in the trivial kernel of the representation of $G$ on $V$. Using an obvious notation, we have $A_{B}=d\left(\zeta_{1}+\cdots+\zeta_{x}\right)$; where $d \in \mathbb{N}$ and the $\zeta_{j}$ are pairwise non-isomorphic one-dimensional representations of $B$ over $\overline{\mathbb{F}}$. Therefore, if $\omega$ is an eigenvalue of $A(g), g \in B$, with $\omega \neq 1$, then $\omega^{-1}$ occurs with multiplicity $d$ in $A(g)$ as well. Let $\zeta_{1}(\mathrm{~g})=\omega \neq 1$. 1. Define $\zeta^{-}$via $\zeta^{-}(b)=\left(\zeta_{1}(b)\right)^{-1}$, any $b \in B$. Since $B$ is abelian of odd order, $\zeta^{-}$is a one-dimensional representation of $B$ over $\mathbb{F}$ with $\zeta^{-} \neq \zeta_{1}$. Now $A_{B}(b)=$ $A_{B}\left(b^{-1}\right)$ for all $b \in B$. Thus by applying an orthogonality relation it follows that $\zeta^{-}$ occurs in $A_{B}$, say $\zeta^{-}=\zeta_{2}$. Now observe that $x=\mid N$ : (inertia group of $\zeta_{i}$ in $N$ ) $\mid$ divides $|N / F(N)|$, as (inertia group of $\zeta_{i}$ in $\left.N\right) \supseteq F(N)$. As $|N / F(N)|$ is odd by assumption, this means that at least one $\zeta_{i}$ is the trivial character of $B$ over $\mathbb{F}$, say $\zeta_{y}=1_{B}$. Then immediately it holds that $B$ is trivially represented on $V$ for we know from Clifford's Theorem that all the $\zeta$ 's are $N$-conjugated to each other. However, as $V_{N}=2 U, U$ is faithful as $\mathbb{F} N$-module and we have a contradiction.

For the convenience of the reader we repeat the definition of a monoprimary module.

Definition. Let $K$ be a finite field. Let $V$ be a non-singular symplectic $K G$-module for the finite group $G$. Then $V$ is called monoprimary if it is a direct sum of pairwise non-isomorphic, self-dual, irreducible $K G$-modules.

There are places in the literature, such as $[2,7,8,9,11]$, where the property of being a monoprimary module yields results in the theory of $M$-groups. As a tool for applications one would like to know a theorem like "If $N \unlhd \underline{G}, V$ a monoprimary $K G$ module, then $V_{N}$ is a monoprimary $K N$-module". This is certainly not true in its full generality. In this respect we can prove such a theorem in a particular case.

Theorem $C$. Let $G$ be a finite group, $N \supsetneqq G, G / N$ solvable of odd order. Suppose that every prime divisor of $|G / N|$ divides $|\mathbb{F}|-1$ with $\mathbb{F}$ a finite field. Let $V$ be a monoprimary FG-module. Then $V$ is also monoprimary as $\mathbb{F} N$-module. 


\section{CLIFFORD'S THEOREM AND RAMIFICATION INDICES}

Proof. In order to prove the theorem, we can clearly restrict outselves to the case $|G / N|=q, q$ odd prime. Next we argue that it suffices to assume that $V$ is an irreducible $\mathbb{E} G$-module. Namely, let $V=A_{1} \perp \cdots \perp A_{t}, A_{i} ¥ A_{j}$ as $\mathbb{F} G$-modules when $i \neq j$, the $A$ 's nonsingular symplectic irreducible $\mathbb{F} G$-modules. Since $\mathbb{F}$ is a splitting field for $G / N$ with (char $\mathbb{F}) \chi|G / N|$, it is possible to apply Lemma (3.4) of [8]. In that lemma it is proved that if $\left.A_{i}\right|_{N}$ and $\left.A_{j}\right|_{N}$ have a common irreducible constituent in the Clifford sense, $A_{i} \cong A_{j} \lambda$ for some one-dimensional $\mathbb{F}$-character $\lambda$ of $G / N$. Now $A_{i}$ and $A_{j}$ are both selfdual. Then, since $\lambda$ is a $\mathbb{F}$-character of $G$ of odd multiplicative order, Proposition (3.7) of [8] implies that $A_{j} \lambda \cong A_{j}$, whence $i=j$. Thus from now on, we assume that $V$ is an irreducible non-singular symplectic $\mathbb{F} G$-module. The proof of the theorem follows now from a variation of Theorem A, to be called Theorem D.

The proof of the following Theorem $\mathrm{D}$ can be regarded as a specialization of the proof of Theorem A, but there are some subtleties in it. As mentioned in the Introduction, the statement of Theorem D resembles that of the analogous statement made in the proof of Theorem (3.1) of [8].

Theorem D. Let $G$ be a finite group. Suppose $G$ admits an irreducible non-singular symplectic $\mathbb{F} G$-module $V$ for a certain finite field $\mathbb{F}$. Let $N \triangleleft G,|G / N|=q, q$ odd prime. Assume $q$ divides $|\mathbb{F}|-1$. Then precisely one of the following statements holds.

(1) $V_{N}=U_{1} \perp \cdots \perp U_{q}, U_{i} ¥ U_{j}$ if $i \neq j$, the $U_{t}$ are irreducible non-singular symplectic FN-submodules of $V_{N}$.

(2) $V_{N}$ is an irreducible (whence non-singular symplectic) $\mathbb{F} N$-module:

Proof. It is clear that we can assume that

$$
V_{N} \text { is homogeneous, say } V_{N}=e U \text {; }
$$

just follow part (A) of the proof of Theorem $A$. Let $K$ be the field defined in the beginning of part (B) of the proof of Theorem A. Again we have

$$
V \bigotimes_{\mathbf{F}} K=M_{1} \perp \cdots \perp M_{a} \perp\left(M_{a+1} \dot{+} M_{a+1}^{*}\right) \perp \cdots \perp\left(M_{a+u} \dot{+} M_{a+u}^{*}\right) .
$$

In this equality $(\beta)$ all the written $M$ 's and $M^{*}$ 's are all pairwise non-isomorphic and they are all galois conjugated to each other. Next we split up.

Assume $u=0$, i.e. $V \bigotimes_{\mathrm{F}} K=M_{1} \perp \cdots \perp M_{a}$. Hence $V \bigotimes_{\mathrm{F}} K$ is monoprimary. Now $\left(V \bigotimes_{F} K\right)_{N}$ is monoprimary as soon as we have proved that each $\left.M_{i}\right|_{N}$ is monoprimary. Indeed, $|\mathbb{F}|-1$ divides $|K|-1$, so $q$ divides $|K|-1$ and we can use Lemma (3.4) and Proposition (3.7) of [8] again. Observe however that $\left.M_{i}\right|_{N}$ satisfies either statement of Theorem $\mathrm{D}$. It holds because $M_{i}$ is an absolutely irreducible $K G$-module, being also non-singular symplectic, following Theorem VII.9.18 of [4]. Hence, as $\left(V \otimes_{F} K\right)_{N} \cong$ $V_{N} \bigotimes_{\mathrm{F}} K \cong(e U) \bigotimes_{\mathrm{F}} K \cong e\left(U \bigotimes_{\mathrm{F}} K\right)$, the Krull-Schmidt Theorem immediately gives $e=1$.

Let $u \geqq 1$. Just as it is done in part (B.2. $\beta$ ) of the proof of Theorem A, we have $V \otimes_{\mathrm{F}} K=\left(M_{1} \dot{+} M_{1}^{*}\right) \perp \cdots \perp\left(M_{u} \dot{+} M_{u}^{*}\right)$. Again there is here a field tower $F \subseteq F_{r} \subset F_{r 2}=K$ 
such that $V \bigotimes_{\mathbf{F}} \mathbb{F}_{r}=L_{1} \perp \cdots \perp L_{u}$, that is, $V \bigotimes_{F} \mathbb{F}_{r}$ is monoprimary. As $|\mathbb{F}|-1$ divides $\left|\mathbb{F}_{r}\right|-1$, it holds that $q|| \mathbb{F}_{r} \mid-1$. By Lemma (3.4) and Proposition (3.7) of [8], $\left(V \bigotimes_{F} \mathbb{F}_{r}\right)_{N}$ is monoprimary as soon as each $\left.L_{i}\right|_{N}$ is monoprimary. Having achieved that result, the Krull-Schmidt Theorem gives $e=1$ in the relation .

$$
\left(V \bigotimes_{F} \mathbb{F}_{r}\right)_{N} \cong(e U) \bigotimes_{F} \mathbb{F}_{r} \cong e\left(U \bigotimes_{F} \mathbb{F}_{r}\right) \cong\left(L_{1} \perp \cdots \perp L_{u}\right)_{N}
$$

We now pick such a $\mathbb{F}_{r} G$-module $L_{i}$, we call it $L$. Thus $L$ is a non-singular symplectic irreducible $\mathbb{F}_{r} G$-module with $L \bigotimes_{\mathrm{Fr}} K=M+M^{*}$ and these $K G$-modules $M$ and $M^{*}$ are dual to each other. Besides that, they are absolutely irreducible non-isomorphic isotropic $K G$-modules.

Next assume that $M_{N}$ and $\left.M^{*}\right|_{N}$ have a common irreducible constituent in the Clifford sense. Then $M^{*} \cong M \mu$ for some one-dimensional $K$-character $\mu$ of $G / N$. As $q|| \mathbb{F}_{r} \mid-1$ and $\left(\left|\mathbb{F}_{r}\right|-1\right) \mid(|K|-1)$, we see that $\mu$ is in fact a one-dimensional $\mathbb{F}_{r}$-character of $G / N$ of odd order. Since $M^{*} \cong M^{\sigma}$ for some $\sigma \in \operatorname{Gal}\left(K / \mathbb{F}_{r}\right)$ with $\sigma^{2}=1, M$ is a socalled weakly self-dual module over $\mathbb{F}_{r}$, see Definition (3.6) of [8]. As both $M$ and $M \mu \cong M^{*}$ are weakly self-dual over $\mathbb{F}_{r}$, Proposition (3.7) of [8] yields $M \mu \cong M$. Thus we have a contradiction and so $M_{N}$ and $\left.M^{*}\right|_{N}$ do not have common irreducible constituents.

Hence, applying the Krull-Schmidt Theorem and the fact that $M$ and $M^{*}$ are absolutely irreducible $K G$-modules, $\left(L \bigotimes_{F_{r}} K\right)_{N}$ decomposes into a direct sum of pairwise non-isomorphic irreducible $K N$-modules. Then $L_{N}$ must also decompose in a direct sum of pairwise non-isomorphic irreducible $\mathbb{F}_{r} N$-modules. Now, go to the written text in the proof of Theorem $A$ in case (A) for the non-singular symplectic $\mathbb{F}_{r} G$-module $L$ instead of the $\mathbb{F} G$-module $V$ written there. It follows then, that $L_{N}$ is monoprimary.

Therefore $\left(V \bigotimes_{\mathrm{F}} \mathbb{F}_{r}\right)_{N}$ is monoprimary as we have seen. Hence the Krull-Schmidt Theorem applied to $\left(V \bigotimes_{\mathrm{F}} \mathbb{F}_{r}\right)_{N} \cong e\left(U \bigotimes_{\mathrm{F}} \mathbb{F}_{r}\right)$ yields $e=1$.

In the next theorem we show that the value of the ramification index $e$ is restricted in the case that we work with modules over a finite field.

Theorem E. Let $G$ be a finite group, $N \triangleleft G,|G / N|=q, q$ some prime integer. Assume $V$ is an irreducible $\mathbb{F} G$-module for a certain finite field $\mathbb{F}$. Suppose that $V_{N}=e U$, that is, if $V$ is considered as $\mathbb{F} N$-module, it is a direct sum of $e$ isomorphic copies of the irreducible $\mathbb{F} N$ submodule $U$ of $V_{N}$. Then $e=1$ or $e=q$ or e divides $q-1$.

Proof. Let char $\mathbb{F}=p$. We can assume that $q \neq p$ for otherwise Green's Theorem VII.9.19 of [4] gives $e=1$. Hence let $q \neq p$. By Theorem VII.2.6 of [4] there exists a finite field $K$ containing $F$ such that $K$ is a splitting field for $G$, for $N$ and for $G / N$ all together. Consider $V \bigotimes_{\mathrm{r}} K$. Then, for suitable integers $u$ and $s$, we have the following decompositions into irreducible $K G$-modules $R_{j}$ and irreducible $K N$-modules $T_{j}$ :

$$
V \bigotimes_{F} K=R_{1}+\cdots+R_{u}, \quad(e U) \bigotimes_{F} K \cong e(U \underset{F}{\bigotimes} K)=e\left(T_{1} \dot{+} \cdots+T_{s}\right) .
$$


Since Schur indices for modules over finite fields are all equal to one ([4], VII.1.16.e), it follows that the $R_{i}$ are pairwise non-isomorphic absolutely irreducible $K G$-modules affording characters which are galois conjugated to each other, see $[5,9.21]$. The same statement holds for the $K N$-modules $T_{i}$.

(1) Let $\left.R_{1}\right|_{N}$ be not homogeneous. Let $W$ be an irreducible constituent of the $K N$ module $\left.R_{1}\right|_{N}$. Then Clifford's Theorem yields $R_{1} \cong W \bigotimes_{K N} K G$, that is, $R_{1}$ is induced by $W$. Moreover, $\left.R_{1}\right|_{N}$ is the direct sum of $q$ pairwise non-isomorphic $G$-conjugated $K N$ submodules. All these $K N$-modules are absolutely irreducible. From the Krull-Schmidt Theorem we see that some $T_{j}$ is isomorphic to $W$ as $K N$-modules. Since all the $T_{i}$ have the same $K$-dimension, it follows that, after an eventual renumbering, $q u=s, e=1$, $R_{i} \cong T_{i} \bigotimes_{K N} K G$. Notice that it is implicitly used here that if some $\left.R_{i}\right|_{N}$ happens to be homogeneous that $\left.R_{i}\right|_{N}$ is irreducible as $K N$-module, by [4, VII.9.19 and VII.9.18], just by the splitting field property of $K$. Thus in fact all $\left.R_{i}\right|_{N}$ are here not homogeneous.

(2) Suppose now that all $\left.R_{i}\right|_{N}$ are homogeneous. Then [4, VII.9.18] implies that all the $\left.R_{i}\right|_{N}$ are absolutely irreducible $K N$-modules. Let $D=\left\{R_{1}, \ldots, R_{u}\right\}$. Let $Y$ be an $\mathbb{F}(\chi)$ submodule of $V \bigotimes_{F} F(\chi)$, where $\chi$ is the trace function of $R_{i}$ (the field $F(\chi)$ does not depend on the index $i$, by $[5,9.21 . c])$. Then $Y \bigotimes_{\mathrm{F}(x)} K$ is an absolutely irreducible $K G$ module isomorphic as $K G$-module to a member of $D$. See $[5,9.21$.e]. So we have

$$
V \bigotimes_{F} \mathbb{F}(\chi)=S_{1}+\cdots+S_{u},
$$

where, say, $R_{i} \cong S_{i} \bigotimes_{\mathbb{F}(x)} K$, and where, by the Deuring-Noether Theorem $[5,9.7], S_{i} ¥ S_{j}$ if $i \neq j$, as $\mathbb{F}(\chi) G$-modules. Observe that any $S_{i}$ is an absolutely irreducible $\mathbb{F}(\chi) G$-module. Consider an irreducible constituent $Z$ of $\left.S_{i}\right|_{N}$. By the Krull-Schmidt Theorem it is isomorphic as $\mathbb{F}(\chi) N$-module to some irreducible constituent of $U \bigotimes_{F} F(\chi)$. Then some irreducible constituent of $Z \bigotimes_{\mathrm{F}(x)} K$ is, by Krull-Schmidt again, isomorphic to some irreducible constituent of $U \bigotimes_{\mathrm{F}} K \cong\left(U \bigotimes_{\mathrm{F}} \mathrm{F}(\chi)\right) \bigotimes_{\mathrm{F}(x)} K$. That last constituent must be isomorphic to one of the $\left.R_{i}\right|_{N}$. By comparison of dimensions it now holds that $\left.S_{i}\right|_{N}$ is an absolutely irreducible $\mathbb{F}(\chi) N$-module for any $i$. Notice now that $u=[\mathbb{F}(\chi): \mathbb{F}]=$ $|\mathrm{Gal}(\mathbb{F}(\chi) / \mathbb{F})|$ and that $\mathrm{Gal}(\mathbb{F}(\chi) / \mathbb{F})$ is cyclic, generated by the Frobenius automorphism $x \mapsto x^{b}$, where $b=|\mathbb{F}|$, any $x \in \mathbb{F}(\chi)$. We have $e t=u$, where $t$ is just the number of all the isomorphy types of the irreducible $\mathbb{F}(\chi) N$-submodules of $U \bigotimes_{\mathbb{F}} \mathbb{F}(\chi)$. Such a module is isomorphic to some $\left.S_{i}\right|_{N}$. Suppose from now on that $e \geqq 2$ and let $\bar{D}=\left\{S_{1}, \ldots, S_{u}\right\}$. Hence there are $A, B \in \bar{D}$ with $A \neq B$ with $A_{N} \cong B_{N}=\left.S_{1}\right|_{N}$, say. Observe that $\bar{D}=$ $\left\{A^{\tau} \mid \tau \in \operatorname{Gal}(\mathbb{F}(\chi) / \mathbb{F})\right\}$. Let $\Pi=\left\{\left.\sigma \in \operatorname{Gal}(\mathbb{F}(\chi) / \mathbb{F})\left|A^{\sigma}\right|_{N} \cong S_{1}\right|_{N}\right\}$. So $\Pi \neq\{1\}$. Hence, if $\sigma \in \Pi$, there exists by [4, VII.9.13], a unique one-dimensional $\mathbb{F}(\chi) G$-representation $\Lambda_{\sigma}$, depending on $\sigma \in \Pi$ and with $N$ acting trivially on $\Lambda_{\sigma}$, such that $A^{\sigma} \cong A \bigotimes_{\mathrm{F}(x)} \Lambda_{\sigma}$. Therefore if $\alpha, \beta \in \Pi$,

$$
\begin{aligned}
& \left(A^{\alpha}\right)^{\beta} \cong\left(A \underset{f(x)}{\bigotimes_{\alpha} \Lambda_{\alpha}} \cong A^{\beta} \bigotimes_{f(x)}\left(\Lambda_{\alpha}\right)^{\beta}\right. \\
& \cong\left(A \bigotimes_{f(x)} \Lambda_{\beta}\right) \underset{f(x)}{\otimes}\left(\Lambda_{\alpha}\right)^{\beta} \text {. }
\end{aligned}
$$

So, if $\alpha, \beta \in \Pi$ then $\alpha \beta \in \Pi$. Hence $\Pi$ is a subgroup of the cyclic group $\operatorname{Gal}(\mathbb{F}(\chi) / \mathbb{F})$. 
Let $\Pi=\langle\gamma\rangle$ and let $\mathbb{F}_{\theta^{j}}$ be the invariant field of $\langle\gamma\rangle$. Notice that $1=(b, q)$. It follows that $E:=\left\{A, A^{\gamma}, \ldots A^{\gamma^{|\gamma|-1}}\right]$ is precisely the subset of $D$ consisting of those members which are isomorphic to $\left.S_{1}\right|_{N}$ when they are realized as $\mathbb{F}(\chi) N$-modules. Notice $|E|=|\gamma|$. Let $\phi \in \mathrm{Gal}(\mathbb{F}(\chi) / \mathbb{F})$ be arbitrary. Then $A^{\phi} \in \bar{D}$ and.each member of $\bar{D}$ is of this form. It follows that (with $\left.\Lambda=\Lambda_{y}\right),\left(A^{\phi}\right)^{\gamma^{i}}=\left(A^{\gamma^{i}}\right)^{\phi} \cong\left(A \bigotimes_{\mathrm{F}(x)} \Lambda^{f}\right)^{\phi} \cong A^{\phi} \bigotimes_{\mathrm{F}(x)}\left(\Lambda^{f}\right)^{\phi}$, where

with

$$
\Lambda^{f} \cong \underbrace{\Lambda \bigotimes_{\mathrm{F}(x)} \Lambda \bigotimes_{\mathrm{F}(x)} \cdots \bigotimes_{\mathrm{F}(x)} \Lambda}_{f \text {-times }}
$$

$$
f=\frac{\left(b^{j}\right)^{i}-1}{b^{j}-1}
$$

Hence $\left.\left.\left(A^{\phi}\right)^{\gamma^{i}}\right|_{N} \cong A^{\phi}\right|_{N}, i=1, \ldots,|\gamma|-1$. Therefore we see that $|\gamma| t=u$. So $e=|\gamma|$. It follows that $A \cong A^{\gamma^{e}} \cong A \bigotimes_{\mathrm{F}(x)} \Lambda^{h}$ with

$$
h=\frac{\left(b^{j}\right)^{e}-1}{b^{j}-1}
$$

Now, by [4, VII.9.12.c], $\Lambda^{h}=I$, the trivial one-dimensional $\mathbb{F}(\chi) G$-module. As $\Lambda$ has order $q$, we have $q \mid h$. If now $q$ divides

$$
\frac{\left(b^{j}\right)^{a}-1}{b^{j}-1}
$$

for some $a \in\{1, \ldots, e-1\}$, then $A^{\gamma^{a}} \cong A$ and so $|E| \leqq a<e=|E|$, a contradiction. Hence $q$ does not divide

$$
\frac{\left(b^{j}\right)^{a}-1}{b^{j}-1}
$$

if $a \in\{1, \ldots, e-1\}$. Next, if $q$ does not divide $b^{j}-1$, then it follows that $e$ is precisely equal to the order of $b^{j}$ modulo $q$. By Fermat's Theorem $\left(b^{j}\right)^{q-1} \equiv 1(\bmod q)$, whence $e \mid q-1$. Therefore assume now that $q \mid b^{j}-1$. This means that

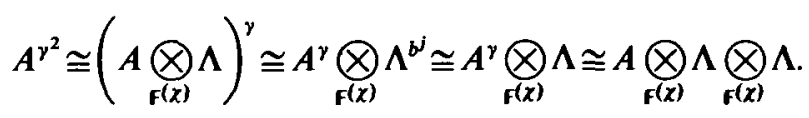

Hence

$$
A^{\gamma^{i}} \cong A \bigotimes_{\mathbf{F}(x)}^{\otimes} \underbrace{(\Lambda \otimes \cdots \otimes \Lambda)}_{i \text {-times }}
$$

Therefore $|\gamma|=q$, and then $e=|\gamma|=q$. This finishes the proof of the theorem. 


\section{REFERENCES}

1. T. R. Berger, Representation theory and solvable groups: length type problems, Proc. Sympos. Pure Math. 37 (1980), 431-441.

2. E. C. DADE, Monomial characters and normal subgroups, Math. Z. 178 (1981), 401-420.

3. B. HuPPERT, Endliche Gruppen I (Springer Verlag, Berlin-Heidelberg-New York, 1967).

4. B. Huppert and N. Blackburn, Finite Groups II (Springer Verlag, Berlin-Heidelberg-New York, 1982).

5. I. M. IsaAcs, Character Theory of Finite Groups (Academic Press, New York-London, 1976).

6. I. M. IsaAcs, Characters of solvable groups, Proc. Sympos. Pure Math. 37 (1980), 377-384.

7. I. M. IsaACs, Primitive characters, normal subgroups and M-groups, Math. Z. 177 (1981), 267-284.

8. I. M. IsaACs, Abelian normal subgroups of M-groups, Math. Z. 182 (1983), 205-221.

9. A. E. Parks, Nilpotent by supersolvable M-groups, Canad. J. Math. 37 (1985), 934-962.

10. R. W. van DeR WaAlL, Minimal non-M-groups, Indag. Math. 42 (1980), 93-106.

11. R. W. van Der WaAll, Minimal non-M-groups III, Indag. Math. 45 (1983), 483-492.

12. R. W. van DER WAall and N. S. HeKster, Irreducible constituents of induced monomial characters, J. Algebra, to appear.

13. W. WILLEMS, Induzierte und eingeschränkte Moduln über Gruppenringen (Diplomarbeit, Mainz, 1973).

Mathematisch InstituUt

Universiteit Van AMSTERDAM

ROETERSSTRAAT 15

1018 WB AMSTERDAM

The NetherLands 